\section{Response to Wald et al}

To the Editor: I would like to thank Professor Wald and his team ${ }^{1}$ for their article adding to the evidence base for the prenatal analysis of plasma DNA, also called noninvasive prenatal testing in the UK or screening (NIPT or NIPS), to screen for trisomies 21,18 , and 13. NIPS has previously been shown to be a superior test compared with combined screening methods involving serum markers and ultrasound scanning. ${ }^{2}$ Wald et al. confirm the superiority of NIPS over the combined test when implemented in routine hospitalbased service in the United Kingdom. Furthermore, in the discussion section, the authors extrapolate findings to discuss the advantages of the reflex pathway over the contingent pathway.

Studies have demonstrated that NIPS yields the most accurate results in populations of high prevalence. ${ }^{2}$ Therefore, in England and Wales, National Health Service commissioners have opted for an evaluative rollout of NIPS as a second-stage test in a population of pregnancies already found to have a higher chance of being affected by a trisomy by conventional methods (a "combined test" first stage).

Parents will be given the results of their first-stage test and then supported through making decisions about further testing. This is described as "contingent" testing in the paper by Wald et al. ${ }^{1}$ and is compared with their preferred "reflex" testing model. Both models use plasma-free DNA technology, but in the reflex pathway blood is taken at the first-stage appointment and is tested only when results from this stage are known and a pregnancy with a high chance of being affected by a trisomy is identified. First-stage results are withheld from parents until second-stage testing is complete.

As a member of the working group for the Nuffield Council on Bioethics report "Non-Invasive Prenatal Testing: Ethical Issues,"3 I should like to share my experience and add a personal note of caution about extrapolating too far in this complicated area. In our evidence-gathering exercise we consulted health-care professionals, regulators, parents, the interested public, and third-sector organizations. Concerns were raised about the reflex pathway because of the jump between first and second stages without the opportunity to offer time, support, and counseling to parents and to check informed consent for NIPS in the light of new evidence. ${ }^{3}$

Wald et al. indicate a potential for cost saving resulting from "the reduction in the number of invasive diagnostic tests needed and the reduced need for patient counseling associated with the two-step approach." This is a seductive argument. However, cost and clinical effectiveness are only part of the screening context.
When comparing any NIPS pathway to conventional screening, there is an expectation of a reduction in the number of invasive diagnostic tests because of its greater specificity. ${ }^{2}$ In the context of a contingent versus a reflex model, the authors cite a report that $18 \%$ of women recalled after the first stage in a contingent model proceeded straight to diagnostic testing at the risk of increasing the number of diagnostic tests and the falsepositive rate when compared with reflex testing. ${ }^{1}$

A different framing is that after the first stage in the contingent model, parents were free to decline to consent to NIPS and opt for the certainty of a diagnostic procedure. Nearly a fifth of parents chose this route. When parents understand the consequences and benefits of NIPS testing and have capacity to make this decision, there is value in exercising their reproductive autonomy. Exercise of autonomous, informed reproductive choices was one of the central principles put forward by the Nuffield Council. ${ }^{3}$

The resource advantage in the reflex screening pathway requires one fewer visit to the clinic for blood taking and counseling than the contingent method. However, whether this benefit would be maintained for the wider health system is not examined. It would be necessary to capture resource use in antenatal and other services by parents following both pathways to determine whether the reflex screening model reduced demand overall or shifted it elsewhere.

The authors advance an argument for the reflex pathway on the grounds of compassion, that parents are made acutely anxious by being recalled following first-stage results and that this is avoided by withholding this information from parents until the NIPS result is available to further inform their decision.

According to Wald et al.,1 "We can be confident that the reflex DNA screening strategy benefits women by reducing the chance that they will be made acutely anxious. Measuring anxiety levels directly in such circumstances is, in our view, neither appropriate nor necessary; imparting potentially distressing information when this can be completely avoided is self-evidently of benefit."

There are several points to raise. First, talking only about women immediately removes male partners from both the decision-making process and experiencing the consequences of testing.

Second, if it is "neither appropriate nor necessary" to discover how anxious parents will be in this circumstance, how will we know if they are more or less anxious or distressed overall by the absence of this stage? The opportunity of an interim discussion will allow a couple time for preparation and exploration of their personal needs for information and support. No studies have yet sought to answer this question with a direct or indirect comparison of the two NIPS pathways under discussion. 
Third, in medicine, there has been a move away from a paternalistic model where clinicians do what they feel is best for the patient albeit with the most compassionate of intentions. To withhold information that formerly parents would have been given routinely, that may aid their decision making, and that current evidence shows would be used by parents is worthy of further consideration.

Finally, I would challenge in this extremely complicated and emotive area of medicine whether anything was so certain to be "self-evidently of benefit" without further evidence. Until we have assessed the value of a two-stage contingent model balanced against the one-stage reflex model this remains uncertain.

\section{DISCLOSURE}

The author declares no conflict of interest.
Clare-Louise Walker, $M B C h B^{1}$

${ }^{1}$ Division of Health Sciences, University of Warwick, Warwick Medical School, Coventry, UK. Correspondence: Clare-Louise Walker

(c.walker.2@warwick.ac.uk)

\section{REFERENCES}

1. Wald NJ, Huttly WJ, Bestwick JP, et al. Prenatal reflex DNA screening for trisomies 21, 18, and 13. Genet Med; e-pub ahead of print 9 November 2017.

2. Chitty LS, Wright D, Hill M, et al. Uptake, outcomes, and costs of implementing non-invasive prenatal testing for Down's syndrome into NHS maternity care: prospective cohort study in eight diverse maternity units. BMJ 2016;354:i3426.

3. Nuffield Council on Bioethics. Non-invasive prenatal testing: ethical issues. Nuffield Council on Bioethics: London, 2017.

Advance online publication 11 January 2018. doi:10.1038/gim.2017.252 